\title{
COFFEE AND NONALCOHOLIC FATTY LIVER DISEASE: A REVIEW
}

\author{
Grzegorz Nikrandt, Agata Chmurzynska ${ }^{凶}$ \\ Institute of Human Nutrition and Dietetics, Poznań University of Life Sciences \\ Wojska Polskiego 31, 60-624 Poznań, Poland
}

\begin{abstract}
Nonalcoholic fatty liver disease (NAFLD) is widely prevalent globally and has no effective treatment. Coffee is one of the most popular beverages in the world and can therefore have a significant impact on public health on account of its health-promoting properties. Evidence from observational, clinical, and animal studies suggests that coffee may play an important role in human health. This article summarizes the effects of coffee on liver health, especially on nonalcoholic fatty liver disease (NAFLD) and its progression: liver fibrosis, cirrhosis and hepatocellular carcinoma. In addition, this article describes the pathogenesis, prevalence, diagnosis, and nutrition guidelines relating to NAFLD. Possible mechanisms responsible for the effects of coffee on the liver are also suggested.
\end{abstract}

Keywords: coffee, caffeine, NAFLD, liver fibrosis, liver cirrhosis, hepatocellular carcinoma

\section{INTRODUCTION}

As a result of the global increase in obesity, nonalcoholic fatty liver disease (NAFLD) has become one of the most common liver diseases in the world, among both adults and children. It is estimated that NAFLD affects about a quarter of the global population, and usually coexists with other metabolic disorders. This disease is progressive, and its most dangerous complication is hepatocellular carcinoma. The above suggests that NAFLD is a serious public health problem with high associated treatment costs; it is therefore necessary to seek effective methods of pharmacotherapy, diet therapy, and prevention of this disease (McCullough, 2006; Younossi et al., 2016).

\section{NAFLD: PROLIFERATION, PATHOGENESIS, AND DIAGNOSIS}

\section{Proliferation}

The prevalence of NAFLD in the world is estimated at $25 \%$, with the highest incidence observed in the
Middle East (31.79\%), South America (30.45\%), and Asia (27.37\%), and the lowest in Africa (13.48\%). The most common comorbidities with NAFLD include hyperlipidemia $(69.16 \%$ of cases), obesity $(51.34 \%$ of cases), metabolic syndrome (42.54\% of cases), hypertension (39.34\% of cases), and type- 2 diabetes (T2DM; 22.51\% of cases) (Younossi et al., 2016).

\section{Pathogenesis}

The pathogenesis of NAFLD and its complications are complex and not fully understood. This section presents only some of the mechanisms related to the development and progression of this disease. One of the most common causes of NAFLD is obesity, but it can also develop in the course of other diseases, such as congenital disorders of lipoprotein metabolism, lipodystrophy, hyperlipidemia, Anderson's disease, Wilson's disease, inflammatory bowel disease, celiac disease, and viral infection by HCV and HCB. The primary form of NAFLD is associated with obesity or

凶agata.chmurzynska@up.poznan.pl 
metabolic syndrome, while the secondary form is associated with other diseases (McCullough, 2006).

An analysis of data from 186 countries, showing changes in the average value of the body mass index (BMI) for men and women in the period 1975-2014, showed an increase in the average BMI from $21.7 \mathrm{~kg} / \mathrm{m}^{2}$ in 1975 to $24.2 \mathrm{~kg} / \mathrm{m}^{2}$ in 2014 among men, and analogously from $22.1 \mathrm{~kg} / \mathrm{m}^{2}$ to $24.4 \mathrm{~kg} / \mathrm{m}^{2}$ among women. The authors of that study predict that, by $2025,18 \%$ of men worldwide and over $22 \%$ of women will suffer from obesity (NCD..., 2016). However, it should be noted that NAFLD can also affect slim people. An American population study based on data from the Third National Health and Nutrition Examination Survey (NHANES III) looked at 11,613 people, of whom 2185 met the criteria for an NAFLD diagnosis, and 9121 formed the control group. Among the group suffering from NAFLD, 431 were slim (BMI $\left.<25 \mathrm{~kg} / \mathrm{m}^{2}\right)$ and 2061 were overweight or obese $\left(\mathrm{BMI}<25 \mathrm{~kg} / \mathrm{m}^{2}\right)$. The results of the study showed that NAFLD is much more common among obese people than slim people $(28 \%$ vs. $7 \%)$ in the American population. Factors that were found to increase the risk of NAFLD in slim people, compared to a control group of healthy slim people, included Hispanic origin, T2DM, and hypertension (Younossi et al., 2012).

The pathogenesis of NAFLD is not fully understood. There are several hypotheses that attempt to explain its development and progression. Hepatic lipid homeostasis is strictly regulated by a complex system of transcription and cell signaling pathways; hormones, transcription factors, and nuclear receptors participate in its control (Arab et al., 2018). Insulin resistance and obesity are thought to play key roles in the development of NAFLD. Excess adipose tissue leads to a change in its secretory activity, leading to an increase in the production of the adipokines involved in the development of insulin resistance, such as leptin, visfatin, resistin, retinol binding protein type-4 (RBP-4), interleukin 6 (IL-6), and cachexin (TNF- $\alpha$ ), and reduction of adiponectin secretion, preventing the development of this disorder (Stojsavljević et al., 2016). The "Multi-Hit" model of NAFLD pathogenesis suggests that interactions between insulin resistance, adipokine secretion by adipose tissue, the inflammation of adipose tissue, and other less well-understood factors (diet, physical activity, environmental factors, intestinal microbiota dysbiosis, genetic factors, and others) occur at the same time and promote hepatitis (Alam et al., 2016).

An unhealthy lifestyle characterized by low physical activity and a high-calorie diet plays a significant role in the development and progression of NAFLD. Studies suggest that an excessive intake of saturated fatty acids (SFA) and fructose stimulates the accumulation of lipid in the liver, leading the disease to progress towards nonalcoholic fatty hepatitis (NASH), while a diet characterized by a high intake of unsaturated fatty acids, choline, antioxidants, protein, and isoflavones has a protective effect against NAFLD. Another type of NAFLD occurs as a result of folate or choline deficiency, leading to lipid accumulation in the liver through very low density protein (VLDL) formation disorders (Radziejewska et al., 2019).

An imbalance between energy consumption and expenditure leads to the accumulation of fat, producing inflammation, insulin resistance, and the release of increased amounts of free fatty acids into the blood, which in turn produces ectopic fat accumulation in the liver, skeletal muscles, and pancreas (de Wit et al., 2012; Shulman, 2014). The presence of excessive levels of fat in the liver depends mainly on increased lipolysis of triglycerides in adipose tissue, and to a lesser extent on dietary fats and sugars and de novo lipogenesis. The accumulation of lipids in hepatocytes damages the hepatocytes and leads to the progression to NASH (Arab et al., 2018; Donnelly et al., 2005). NASH may affect as much as $5 \%$ of the adult population and $20 \%$ of the obese adult population (Townsend and Newsome, 2016). As a result of hepatocyte damage, the immune and apoptotic pathways are activated, which may suffice to cause cell death. This can be followed by further pathological changes that can lead to fibrosis and subsequent cirrhosis (Hirsova and Gores, 2015; Neuschwander-Tetri, 2010).

Chronic liver function damage associated with the destruction of hepatocytes may lead to their cyclic compensatory regeneration and proliferation, favoring the development of liver cancer. Obesity and hyperinsulinemia also contribute to the development of liver cancer. Hypertrophy of adipose tissue and the resulting inflammation leads to increased secretion of proinflammatory adipokines (TNF- $\alpha$, IL-6, leptin) and reduces the secretion of anti-inflammatory adiponectin. 
As a result, the cell cycle accelerates and the proapoptotic pathways are inhibited, leading to an induction of proliferation, invasion processes, migration, and angiogenesis. Intestinal microbiotic disorders associated with obesity lead to increased lipopolysaccharide translocation (LPS), triggering the inflammatory response through toll-like receptors (TLRs), contributing to inflammatory changes and liver fibrosis. Hyperinsulinemia increases the availability of insulin-like growth factor (IGF-1). The signals generated by insulin and IGF-1 promote the proliferation of cells and inhibit their apoptosis in many types of tissues, including the liver. Increased production of reactive oxygen species (ROS) in liver mitochondria modulates the expression of genes involved in hepatocancerogenesis. Insulin resistance also increases the release of free fatty acids from fat cells into the bloodstream; these are involved in fatty liver progression and generate ROS as a result of lipotoxicity, contributing to deoxyribonucleic acid (DNA) damage (Vanni and Bugianesi, 2014).

\section{Diagnostics}

The basis for suspecting the occurrence of NAFLD in a patient is a finding of steatosis of over $5 \%$ of hepatocytes, using imaging techniques such as ultrasonography or proton magnetic resonance spectroscopy, or by histological techniques. Other causes of steatosis should be excluded, including the use of medicines that may interfere with liver function (e.g., methotrexate, corticosteroids, amiodarone, tamoxifen) or the occurrence of hepatitis $\mathrm{C}$ virus (HCV) or hepatitis $B$ virus (HBV). In addition, the patient's alcohol consumption should be less than $20 \mathrm{~g} /$ day for women and less than $30 \mathrm{~g} /$ day for men. Metabolic syndrome and T2DM should also be screened for using laboratory tests (glucose, glycated hemoglobin, lipidogram); liver enzymes (alanine aminotransferase - ALT, aspartate aminotransferase - AST, gamma-glutamyl transferase - GGT) should be determined to allow a more accurate determination of the severity of the disease. It may also be useful to know about the patient's diet and lifestyle (Leoni et al., 2018; Yki-Järvinen, 2016).

\section{DIETARY RECOMMENDATIONS IN NAFLD}

Patients not suffering from NASH or other NAFLD complications should receive appropriate counseling and lifestyle modification recommendations, including for dietary changes and adequate physical activity, without the introduction of pharmacotherapy. Dietary guidelines for NAFLD include those of the European Association for the Study of the Liver (EASL), the National Institute for Health and Care Excellence (NICE), the Association for the Study of the Liver (AISF), and the American Association for the Study of Liver Diseases (AASLD); these differ slightly in some aspects (Leoni et al., 2018). This work will discuss the EASL recommendations (EASL..., 2016).

Weight loss plays an important role in NAFLD diet therapy. It is recommended that the energy supply be reduced by $500-1000 \mathrm{kcal} / \mathrm{week}$, thus obtaining a weight reduction of about $0.5-1 \mathrm{~kg}$ per week. In overweight and obese people, the goal of caloric restriction is to reduce their weight by $7-10 \%$, facilitating a decrease in liver enzymes and an improvement in the histological image of the liver tissue. In order to reduce weight, it may be helpful to introduce physical activity and cognitive behavioral therapy. Highly processed and fructose-rich foods should be excluded from the diet. The distribution of macronutrients should be in accordance with the recommendations of the Mediterranean diet. Alcohol consumption should be kept low, at no more than $30 \mathrm{~g}$ for men and $20 \mathrm{~g}$ for women daily. Much research has pointed to the hepatoprotective function of coffee, so restrictions on drinking coffee are not recommended (EASL..., 2016; Liu et al., 2015).

Physical activity is recommended in the form of both aerobic and resistance training, at 150-200 minutes per week, divided into 3-5 sessions. The type of training should be adapted to the patient's preferences in order to ensure compliance (EASL..., 2016).

\section{COFFEE IN THE PREVENTION OF NAFLD AND ITS COMPLICATIONS}

\section{Coffee characteristics}

Coffee is one of the most popular drinks in the world. In 2019, Americans drank about 1.63 billion tons of coffee, making the United States the second largest coffee market in the world after Europe (Foreign..., 2019). More and more research points to the preventive properties of coffee against many diseases, and particularly liver diseases (Poole et al., 2017). 
The coffee plant belongs to the family Rubiaceae and genera Coffea. Commercial coffee production is based on two plant species, Coffea arabica, commonly known as Arabica coffee, which accounts for over $60 \%$ of the world's coffee production and Coffea canephora, which is used to produce Robusta coffee and accounts for less than $40 \%$ of the world's coffee production (Farah and Ferreira dos Santos, 2015).

Coffee is a complex mixture of compounds, the amounts of which depend on the type of coffee, its roasting, and its processing. Green coffee beans contain mainly carbohydrates (about 60\% dry mass), including polysaccharides, oligosaccharides, disaccharides, and monosaccharides. The lipid content of green coffee beans accounts for $8-18 \%$ of its dry mass, while the proteins, peptides, and free amino acids in green coffee beans account for $9-16 \%$ of its dry mass. Moreover, green coffee beans are a rich source of polyphenols, which constitute $6-10 \%$ of its dry weight. The main components of green coffee polyphenols are chlorogenics acids (CGAs), with 5-O-caffeoylquinic acid (5-CQA) being the most abundant, and lower amounts of feruloylquinic acids (FQAs) and dicaffeoylquinic acids (di-CQAs) (Ludwig et al., 2014).

Roasting coffee beans at high temperatures leads to chemical reactions between amino acids and carbohydrates, known as Maillard reactions, which result in a number of unique ingredients. The most commonly studied ingredient in roasted coffee is caffeine, but it also contains a number of other bioactive compounds. The most important of these include chlorogenic acids, which are polyphenols, as well as cafestol and kahweol, which are diterpenes, melanoidin, and trigonelline, which are converted to niacin during roasting (Nieber, 2017). Drastic roasting processes may result in an 8-10\% loss of CGAs for every $1 \%$ loss of dry matter (Ludwig et al., 2014). Additionally, roasted coffee contains acrylamide, a product of Mailard reactions, which is likely to be carcinogenic (WHO, 2006).

Recent studies have shown that coffee consumption may play an important role in the prevention of T2DM, neurological diseases, depression, cancer, hepatic injury and cardiovascular disease (De Melo Pereira et al., 2020).

\section{Studies on laboratory animals}

Vitaglione et al. (2010) conducted tests on laboratory rats. The aim of their study was to investigate the effects of adding coffee or its bioactive ingredients to the diet on the development of NAFLD. They found that the concentration of triacylglycerols in rats receiving a high-fat diet (HFD) with the addition of a mixture of coffee polyphenols was significantly higher than in the control group. Significantly lower levels of triacylglycerols were observed in rats receiving the HFD diet with the addition of coffee and the HFD diet with the addition of melanoidins than in rats receiving the HFD diet with water. In addition, a significant increase in ALT was seen in the rats receiving the HFD diet with added water, as compared to the other groups. In rats receiving a diet enriched with HFD and coffee or its components, a significant reduction in the presence of fat droplets and inflammatory infiltrates was seen in histological imaging of the liver. In the group receiving the HFD diet with the addition of coffee, interferon-gamma (IFN- $\gamma$ ) and TNF- $\alpha$ in the liver decreased by $17 \%$ and $42 \%$, respectively, while IL- 6 increased by $26 \%$, compared to the HDF group receiving only water. Clinical trials have strongly focused on the effects of caffeine on health. This study suggests that other bioactive compounds may be responsible for the preventive properties of coffee in the liver.

On the other hand, in the study of rats with HFD-induced NAFLD carried out by Salomone et al. (2014), significant increases were observed in the levels of ALT and triacylglycerols in the blood, and of triacylglycerols in the liver, in rats receiving HFD with water compared to those receiving the standard diet (SD) with water. Blood ALT and triacylglycerols were also reduced in rats receiving HFD with coffee compared to those receiving HFD with water, and a two-fold decrease in liver triacylglycerols was seen in rats receiving HFD plus coffee, as compared to the HFD plus water group. Giving coffee to rats also reduced steatosis, fibrosis, inflammatory infiltrates, and ballooning.

A similar study was also conducted by Fujise et al. (2012) on rats with diethylonitrosoamine (DEN)-induced hepatocancerogenesis. This examined the effects of caffeine and curcumin on the alterations in liver function induced by DEN at weeks 10 and 14 of feeding. Statistically lower AST concentrations were seen in rats receiving a normal diet and caffeine for 
14 days $(0.02 \% \mathrm{w} / \mathrm{v})$ than in those receiving curcumin and water and those receiving normal diet and water for 14 days. In addition, liver histology showed fewer and smaller white nodules in rats receiving a normal diet and caffeine than in those receiving curcumin and water, and in those receiving a normal diet and water. This study demonstrated the effectiveness of caffeine in inhibiting carcinogenic lesions in the livers of rats.

The study of Furtado et al. (2014) on male Wistar rats examined the effects of coffee and caffeine on liver fibrosis and carcinogenesis induced by DEN and carbon tetrachloride $\left(\mathrm{CCl}_{4}\right)$. The rats were divided into four groups of twelve. They received drinking water (G1), regular coffee (G2), instant coffee (G3), or $0.1 \%$ caffeine (G4) ad libitum five days a week. A statistically significant inhibitory effect on the development of liver fibrosis was observed in groups G2 and G4. The results of the study also showed that regular and instant coffee and caffeine inhibit preneoplastic and cancerous changes in the liver.

Together, these studies have thus shown that coffee consumption may protect against liver diseases.

\section{Observational studies}

Observational studies suggest a protective effect of both caffeinated and decaffeinated coffee on the risk of NAFLD and its complications, but so far there have not been many such studies. A summary is shown in Table 1. Wijarnpreecha et al. (2017) conducted a meta-analysis of five studies, showing that regular drinkers of coffee have a $29 \%$ lower risk of NAFLD than nondrinkers, while NAFLD sufferers who regularly drink coffee have a $30 \%$ lower risk of liver fibrosis than those sufferers who do not drink coffee. The meta-analysis has the limitation that few studies were included, although most were of high quality. Poole et al. (2017) reviewed 218 meta-analyses on the health effects of coffee. Compared to nonconsumption, coffee consumption was found to reduce the risk of NAFLD, fibrosis, and liver cirrhosis by $29 \%, 27 \%$, and $39 \%$, respectively. Drinking an additional cup of coffee daily was also associated with a $26 \%$ reduction in mortality from liver cirrhosis.

Chen et al. (2019) conducted a systematic review and dose-dependency analysis of seven epidemiological

Table 1. Associations between coffee consumption and liver diseases

\begin{tabular}{|c|c|c|c|}
\hline Author & Disease & Coffee consumption (comparison) & Main findings \\
\hline \multirow[t]{2}{*}{ Wijarnpreecha et al. (2017) } & NAFLD & regular consumption vs. no consumption & RR decrease by $29 \%$ \\
\hline & liver fibrosis & regular consumption vs. no consumption & RR decrease by $27 \%$ \\
\hline Chen et al. (2019) & NAFLD & 0 vs. $>5$ cups/day & RR decrease by $6 \%$ \\
\hline \multirow[t]{3}{*}{ Setiawan et al. (2015) } & $\mathrm{HCC}$ & 1 cup/d vs. no consumption & RR decrease by $13 \%$ \\
\hline & & 2-3 cups/d vs. no consistency & RR decrease by $38 \%$ \\
\hline & & $\geq 4$ cups/d vs. no consumption & RR decrease by $41 \%$ \\
\hline \multirow[t]{2}{*}{ Liu et al. (2015) } & cirrhosis & coffee vs. non-coffee drinkers & $\mathrm{OR}=0.66$ \\
\hline & fibrosis & coffee vs. non-coffee drinkers & $\mathrm{OR}=0.73$ \\
\hline Kennedy et al. (2016) & cirrhosis & additional 2 cups/day & RR decrease by $54 \%$ \\
\hline \multirow[t]{2}{*}{ Kennedy et al. (2017) } & $\mathrm{HCC}$ & additional 2 cups/day & RR decrease by $34 \%$ \\
\hline & $\mathrm{HCC}$ & additional 2 cups of decaffeinated coffee/day & RR decrease by $14 \%$ \\
\hline \multirow[t]{3}{*}{ Poole et al. (2017) } & NAFLD & coffee vs. non-coffee drinkers & RR decrease by $27 \%$ \\
\hline & fibrosis & coffee vs. non-coffee drinkers & RR decrease by $29 \%$ \\
\hline & cirrhosis & coffee vs. non-coffee drinkers & RR decrease by $39 \%$ \\
\hline
\end{tabular}

$\mathrm{HCC}$ - hepatocellular carcinoma, NAFLD - nonalcoholic fatty liver disease, OR - odds ratio, RR - relative risk. 
studies, of which four were cross-sectional studies, two prospective, and one case-control. The total number of respondents was 54,331, of whom 4,825 were diagnosed with NAFLD. The amount of coffee drunk ranged from zero to more than five cups a day. There was no significant relationship between the occurrence of NAFLD in drinkers of less than 1 cup of coffee, compared to those who drank 1-2 cups, or in those drinking $<1$ cup compared to those drinking over 2 cups. The cumulative relative risk of developing NAFLD, comparing the highest consumption of coffee with the lowest, was 0.94 , suggesting a $6 \%$ decrease with each cup of coffee drunk. A dose-dependency analysis showed an inverse relationship between coffee consumption and the relative risk of NAFLD, with the effects being statistically significant when more than three cups of coffee were consumed per day.

The results of epidemiological studies regarding the relationship between coffee consumption and the risk of developing NAFLD complications suggest that coffee consumption has a preventive effect against NASH, cirrhosis, and liver cancer (HCC). Setiawan et al. (2015) examined the relationship between coffee consumption and the incidence of $\mathrm{HCC}$ and mortality from chronic liver disease based on data from the US Multiethnic Cohort Study, conducted on a population of over 225,000 people. After excluding participants who did not meet the research criteria, a total of 162,022 people from various ethnic backgrounds were included. During the eighteen-year follow-up period, 451 cases of liver cancer and 654 deaths due to chronic liver disease occurred in the study group. The relative risk of developing liver cancer decreased as coffee consumption increased, reaching $0.87,0.62$, and 0.59 for consumption of $1,2-3$, and more than 4 cups of coffee, compared to nondrinkers of coffee. In addition, the risk of developing HCC was at least twice as high among non-Caucasians.

The results of a meta-analysis carried out by Liu et al. (2015) suggest that coffee consumers have a lower risk of developing fibrosis and liver cirrhosis than those who do not consume coffee. Sixteen epidemiological studies, mostly conducted in Western countries, were included in the meta-analysis. Most of the studies included were of good quality. The survey covered 3034 coffee consumers and 132,076 non-coffee drinkers. Seven studies suggested that coffee consumption reduces the risk of liver cirrhosis, but only three showed statistical significance. After excluding two studies that were sources of high heterogeneity, the odds ratio (OR) for the risk of liver cirrhosis among coffee drinkers compared to nondrinkers was 0.66 . The analysis of eight studies showed that the OR for coffee drinkers was 0.73 for the chance of developing liver fibrosis compared to the non-coffee group. Kennedy et al. (2016) came to similar conclusions. The meta-analysis involved nine studies with 432,133 participants, including 1990 cases of cirrhosis. It was shown that consuming an additional two cups of coffee a day reduces the risk of liver cirrhosis by $54 \%$.

Liver cancer is one of the most commonly diagnosed cancers. Among its causes are hepatitis B and C, NAFLD, and excessive alcohol consumption. In addition, NASH may progress to HCC without the presence of cirrhosis. Kennedy et al. (2017) examined sixteen articles containing data from 26 studies, covering a total of 2,279,119 people, including 4730 cases of liver cancer. They showed that an increase in coffee consumption by two cups a day reduces the risk of liver cancer by $35 \%$. A dose-dependency analysis also showed that the risk of HCC among people with cirrhosis can be reduced by over $50 \%$ if they consume five cups of coffee a day, compared to not drinking coffee. In studies where the distinction was made, the consumption of an additional two cups of caffeine-containing coffee reduced the risk of developing HCC by $27 \%$, while that of decaffeinated coffee reduced the risk by $14 \%$. The quality of this research, however, was judged to be 'very low' on the GRADE scale. The World Cancer Research Fund and American Institute for Cancer Research (2018) state in their report that there is strong evidence of the protective effects of coffee against liver cancer.

\section{Effects of green coffee extract on NAFLD}

Some studies showed an impact of green coffee extract (GCE) on NAFLD. Hosseinabadi et al. (2020a) conducted a randomized, double-blind placebo-controlled trial. Patients with NAFLD received 2 capsules/day with $200 \mathrm{mg}$ of GCE for 8 weeks $(n=24)$ or 2 capsules/day of placebo for 8 weeks $(n=24)$. Green coffee extract - GCE supplementation significantly reduced BMI and body weight, serum total cholesterol, serum triglyceride, fasting blood glucose, homeostatic model assessment for insulin resistance (HOMA-IR) and increased serum HDL cholesterol levels compared to the 
placebo group. Moreover, serum leptin level decreased significantly in the GCE group compared to the baseline values set by Hosseinabadi et al. (2020b). These studies suggest that GCE may be useful in NAFLD management.

\section{Mechanisms of coffee's effects on the liver}

Coffee is a complex mixture of chemical compounds whose actions may be complementary or synergistic.
Understanding the mechanisms responsible for coffee's protective effects on the liver is thus difficult. Of particular interest among the mechanisms that have been proposed are those which suggest an effect on inflammation markers, a reduction in insulin resistance, or antifibrotic and antitumor activity (Alferink et al., 2018; Bøhn et al., 2014; Chen et al., 2014). These mechanisms are shown in Figure 1.

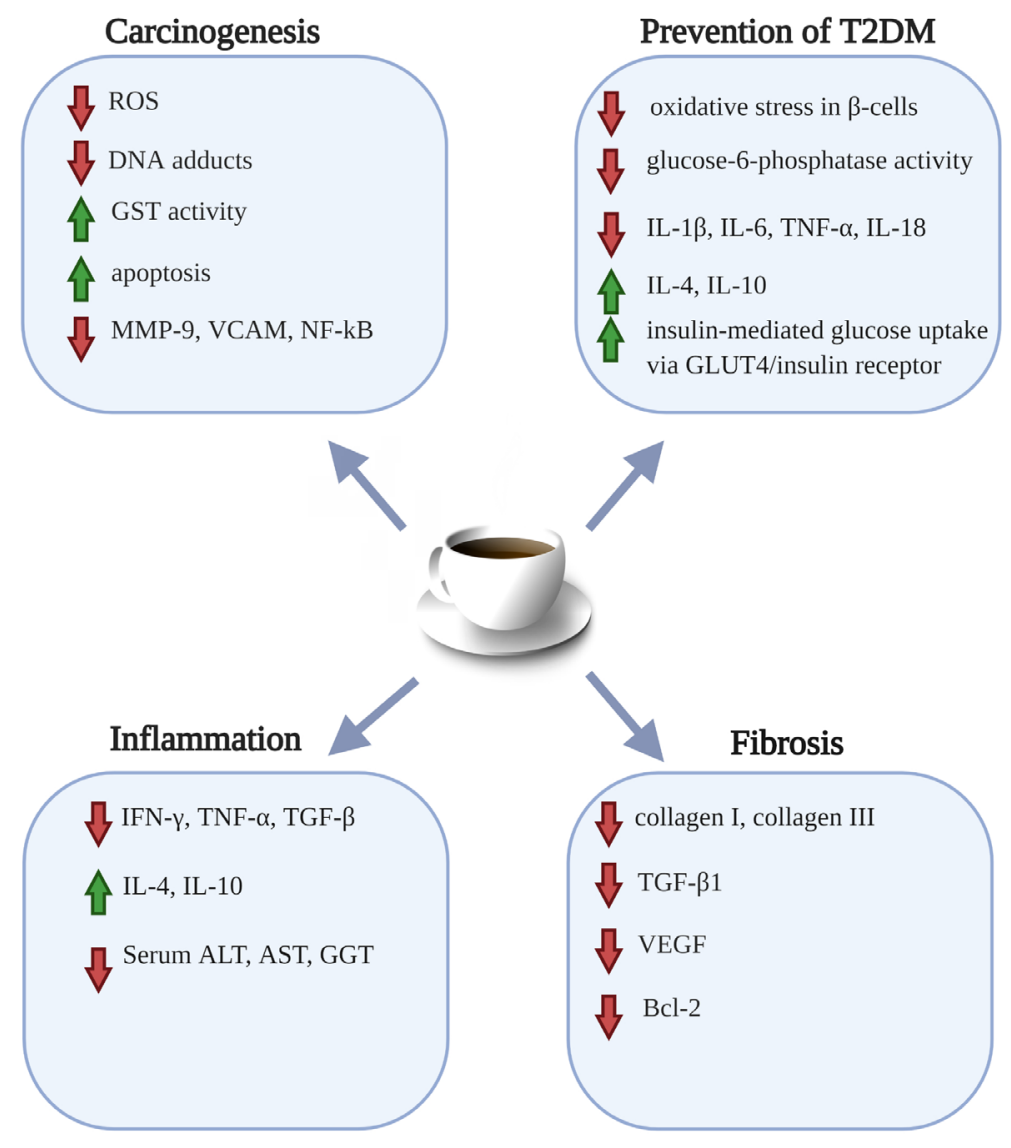

Fig. 1. Probable mechanisms involved in NAFLD prevention by coffee consumption: ALT - alanine transaminase, AST - aspartate transaminase, Bcl-2 - B-cell lymphoma 2, DNA - deoxyribonucleic acid, GGT - gamma-glutamyltransferase, GLUT4 - glucose transporter type 1, GST - glutathione s-transferase, IL-1 $\beta$ - interleukin 1 beta, IL-4 - interleukin 4 , IL6 - interleukin 6, IL-10 - interleukin 10, IL-18 - interleukin 18, INF- $\gamma$ interferon gamma, MMP-9 - matrix metalloproteinase 9, NF-kB - nuclear factor kappa-light-chain-enhancer of activated B cells, ROS - reactive oxygen species, TGF- $\beta$ - tumor growth factor beta, TGF- $\beta 1$ - tumor growth factor beta $1, \mathrm{TNF}-\alpha$ - tumor necrosis factor alpha, VCAM1 - vascular cell adhesion molecule 1, VEGF - vascular endothelial growth factor 
Studies in animal models have shown that the administration of coffee inhibits the progression of NAFLD by inhibiting markers that induce inflammation - such as IFN- $\gamma$, TNF- $\alpha$, and transforming growth factor beta (TGF- $\beta$ ) - while increasing the secretion of the anti-inflammatory cytokines interleukin 4 (IL-4) and interleukin 10 (IL-10) (Honjo et al., 2001; Nakanishi et al., 2000; Ruhl and Everhart, 2005; Shin et al., 2010; Vitaglione et al., 2010).

Regarding insulin resistance and NAFLD, Younossi et al. (2019) have shown that the incidence of NAFLD among T2DM patients is over two times greater than in the general population. In addition, NAFLD may contribute to the development of T2DM by increasing insulin resistance. This may be associated with increased hepatic secretion of diabetogenic proteins, such as fibroblast growth factor 21 (FGF-21) and RBP-4. Ding et al. (2014), in their meta-analysis of prospective studies, showed an inverse relationship between coffee consumption and the risk of T2DM. It is believed that this effect may be due to the following mechanisms underlying the action of coffee: reduced oxidative stress in pancreatic $\beta$ cells, reduced glucose-6-phosphatase activity, reduced proinflammatory secretion, increased secretion of anti-inflammatory factors, and increased insulin-dependent glucose uptake via the glucose transporter type 4 (GLUT-4) receptor and insulin (Akash et al., 2014).

Shin et al. (2010) showed that administering coffee to rats with liver fibrosis induced by carbon tetrachloride reduces the expression of profibrotic factors, such as collagen I, collagen III, TGF- $\beta 1$, vascular endothelial growth factor (VEGF1), and B-cell lymphoma 2 (Bcl-2).

The components of coffee also have anticancer effects, which affect all stages of carcinogenesis. The antioxidant properties of coffee are responsible for its ability to remove the free radicals involved in carcinogenesis. Inhibition of the first stage, initiation, occurs through a reduction of the genotoxicity of carcinogens, via an induction of phase II enzymes, as demonstrated in in vitro studies on human liver cells. Diterpenes, kahweol, and cafestol enhance the ability to repair DNA damage by increasing the expression of proteins responsible for DNA repair, which results in a decrease in the number of DNA adducts. In addition, caffeine and diterpenes increase the activity of glutathione s-transferase (GST), demonstrating antitumor activity by increasing the liver's ability to metabolize xenobiotics. The anti-inflammatory properties of coffee ingredients and their ability to induce apoptosis assist in the removal of damaged cells, inhibiting progression. This is also inhibited through the reduction in synthesis of the proteins involved in angiogenesis, which is necessary for tumor development. Caffeic acid and diterpenes limit tumor progression by inhibiting the compounds involved in tumor growth and metastasis, including metalloproteinases (e.g. matrix metalloproteinase 9, MMP-9), adhesion molecules (such as vascular cell adhesion molecule 1, VCAM1) and nuclear factor kappa-light-chain-enhancer of activated B cells (NF-kB; Alferink et al., 2018; Bøhn et al., 2014).

\section{CONCLUSIONS}

The available data suggest that regular consumption of coffee can reduce the risk of NAFLD and its comorbidities. However, most results come from observational studies and as such there is a need of further intervention studies or animal based studies focused on the mechanisms linking coffee consumption and liver health.

\section{REFERENCES}

Akash, M. S., Rehman, K., Chen, S. (2014). Effects of coffee on type 2 diabetes mellitus. Nutrition, 30(7-8), 755763. http://dx.doi.org/10.1016/j.nut.2013.11.020

Alam, S., Mustafa, G., Alam, M., Ahmad, N. (2016). Insulin resistance in development and progression of nonalcoholic fatty liver disease. World J. Gastroint. Pathophysiol., 7(2), 211-217. http://dx.doi.org/10.4291/wjgp. v7.i2.211

Alferink, L. J., Kiefte-de Jong, J. C., Murad, S. D. (2018). Potential mechanisms underlying the role of coffee in liver health. Semin. Liv. Dis., 38(03), 193-214. http:// dx.doi.org/10.1055/s-0038-1666869

Arab, J. P., Arrese, M., Trauner, M. (2018). Recent insights into the pathogenesis of nonalcoholic fatty liver disease. Ann. Rev. Pathol., 13(1), 321-350. http://dx.doi. org/10.1146/annurev-pathol-020117-043617

Bøhn, S. K., Blomhoff, R., Paur, I. (2014). Coffee and cancer risk, epidemiological evidence, and molecular mechanisms. Mol. Nutr. Food Res., 58(5), 915-930. http:// dx.doi.org/10.1002/mnfr.201300526 
Chen, S., Teoh, N. C., Chitturi, S., Farrell, G. C. (2014). Coffee and non-alcoholic fatty liver disease: Brewing evidence for hepatoprotection? J. Gastroenterol. Hepatol., 29(3), 435-441. http://dx.doi.org/10.1111/jgh.12422

Chen, Y. P., Lu, F. B., Hu, Y. B., Xu, L. M., Zheng, M. H., Hu, E. D. (2019). A systematic review and a dose-response meta-analysis of coffee dose and nonalcoholic fatty liver disease. Clin. Nutr., 38(6), 2552-2557. https://doi.org/10.1016/j.clnu.2018.11.030

De Melo Pereira, G. V., de Carvalho Neto, D. P., Magalhães Júnior, A. I., do Prado, F. G., Pagnoncelli, M. G. B., Karp, S. G., Soccol, C. R. (2020). Chapter three Chemical composition and health properties of coffee and coffee by-products. Adv. Food Nutr. Res., 91, 6596. http://dx.doi.org/10.1016/bs.afnr.2019.10.002

de Wit, N. J., Afman, L. A., Mensink, M., Müller, M. (2012). Phenotyping the effect of diet on non-alcoholic fatty liver disease. J. Hepatol., 57(6), 1370-1373. http://dx.doi. org/10.1016/j.jhep.2012.07.003

Ding, M., Bhupathiraju, S. N., Chen, M., van Dam, R. M., $\mathrm{Hu}$, F. B. (2014). Caffeinated and decaffeinated coffee consumption and risk of type 2 diabetes: a systematic review and a dose-response meta-analysis. Diab. Care, 37(2), 569-586. http://dx.doi.org/10.2337/dc13-1203

Donnelly, K. L., Smith, C. I., Schwarzenberg, S. J., Jessurun, J., Boldt, M. D., Parks, E. J. (2005). Sources of fatty acids stored in liver and secreted via lipoproteins in patients with nonalcoholic fatty liver disease. J. Clin. Invest., 115(5), 1343-1351. http://dx.doi.org/10.1172/ JCI23621

EASL-EASD-EASO Clinical Practice Guidelines for the management of non-alcoholic fatty liver disease (2016). J. Hepatol., 64(6), 1388-1402. http://dx.doi. org/10.1016/j.jhep.2015.11.004

Farah, A., Ferreira dos Santos, T. (2015). The coffee plant and beans. In V. R. Preedy (Ed.), Coffee in health and disease prevention (pp. 5-10). Elsevier. http://dx.doi. org/10.1016/b978-0-12-409517-5.00001-2

Foreign Agriculture Service (2019). 2019/2020 Forecast overview. Coffee: world markets and trade. Washington (DC): USDA.

Fujise, Y., Okano, J. I., Nagahara, T., Abe, R., Imamoto, R., Murawaki, Y. (2012). Preventive effect of caffeine and curcumin on hepatocarcinogenesis in diethylnitrosamine-induced rats. Int. J. Oncol., 40(6), 1779-1788. http://dx.doi.org/10.3892/ijo.2012.1343

Furtado, K. S., Polletini, J., Dias, M. C., Rodrigues, M. A., Barbisan, L. F. (2014). Prevention of rat liver fibrosis and carcinogenesis by coffee and caffeine. Food Chem. Toxicol., 64, 20-26. http://dx.doi.org/10.1016/j. fct.2013.11.011
Hirsova, P., Gores, G. J. (2015). Death receptor-mediated cell death and proinflammatory signaling in nonalcoholic steatohepatitis. Cell. Mol. Gastroenterol. Hepatol., 1(1), 17-27. http://dx.doi.org/10.1016/j.jcmgh.2014.11.005

Honjo, S., Kono, S., Coleman, M. P., Shinchi, K., Sakurai, Y., Todoroki, I., ..., Yoshizawa, N. (2001). Coffee consumption and serum aminotransferases in middle-aged Japanese men. J. Clin. Epidemiol., 54(8), 823-829. http://dx.doi.org/10.1016/s0895-4356(01)00344-4

Hosseinabadi, S., Rafraf, M., Asghari, S., Asghari-Jafarabadi, M., Vojouhi, S. (2020a). Effect of green coffee extract supplementation on serum adiponectin concentration and lipid profile in patients with non-alcoholic fatty liver disease: A randomized, controlled trial. Compl. Ther. Med., 49, 102290. http://dx.doi.org/10.1016/j. ctim. 2019.102290

Hosseinabadi, S., Rafraf, M., Mahmoodzadeh, A., Asghari-Jafarabadi, M., Asghari, S. (2020b). Effects of green coffee extract supplementation on glycemic indexes, leptin, and obesity values in patients with non-alcoholic fatty liver disease. J. Herb. Med., 100340. http://dx.doi. org/10.1016/j.hermed.2020.100340

Kennedy, O. J., Roderick, P., Buchanan, R., Fallowfield, J. A., Hayes, P. C., Parkes, J. (2016). Systematic review with meta-analysis: coffee consumption and the risk of cirrhosis. Aliment. Pharmacol. Ther., 43(5), 562-574. http://dx.doi.org/10.1111/apt.13523

Kennedy, O. J., Roderick, P., Buchanan, R., Fallowfield, J. A., Hayes, P. C., Parkes, J. (2017). Coffee, including caffeinated and decaffeinated coffee, and the risk of hepatocellular carcinoma: a systematic review and doseresponse meta-analysis. BMJ Open, 7(5), e013739. http://dx.doi.org/10.1136/bmjopen-2016-013739

Leoni, S., Tovoli, F., Napoli, L., Serio, I., Ferri, S., Bolondi, L. (2018). Current guidelines for the management of non-alcoholic fatty liver disease: A systematic review with comparative analysis. World J. Gastroenterol., 24(30), 3361-3373. http://dx.doi.org/10.3748/wjg.v24. i30.336

Liu, F., Wang, X., Wu, G., Chen, L., Hu, P., Ren, H., Hu, H. (2015). Coffee consumption decreases risks for hepatic fibrosis and cirrhosis: a meta-analysis. PloS One, 10(11), e0142457. http://dx.doi.org/10.1371/journal. pone. 0142457

Ludwig, I. A., Clifford, M. N., Lean, M. E., Ashihara, H., Crozier, A. (2014). Coffee: biochemistry and potential impact on health. Food Funct., 5(8), 1695-1717. http:// dx.doi.org/10.1039/c4fo00042k

McCullough, A. J. (2006). Pathophysiology of nonalcoholic steatohepatitis. J. Clin. Gastroenterol., 40, 17-29. https://doi.org/10.1097/01.mcg.0000168645.86658.22 
Nakanishi, N., Nakamura, K., Nakajima, K., Suzuki, K., Tatara, K. (2000). Coffee consumption and decreased serum gamma-glutamyltransferase: a study of middleaged Japanese men. Eur. J. Epidemiol., 16(5), 419-23. http://dx.doi.org/10.1023/a:1007683626665

NCD Risk Factor Collaboration (2016). Trends in adult body-mass index in 200 countries from 1975 to 2014: a pooled analysis of 1698 population-based measurement studies with 19.2 million participants. Lancet, 387(10026), 1377-1396. http://dx.doi.org/10.1016/ S0140-6736(16)30054-X

Neuschwander-Tetri, B. A. (2010). Hepatic lipotoxicity and the pathogenesis of nonalcoholic steatohepatitis: the central role of nontriglyceride fatty acid metabolites. Hepatology, 52(2), 774-788. http://dx.doi.org/10.1002/ hep. 23719

Nieber, K. (2017). The impact of coffee on health. Planta Med., 83(16), 1256-1263. http://dx.doi.org/10.1055/ s-0043-115007

Poole, R., Kennedy, O. J., Roderick, P., Fallowfield, J. A., Hayes, P. C., Parkes, J. (2017). Coffee consumption and health: umbrella review of meta-analyses of multiple health outcomes. BMJ, 359, j5024. http://dx.doi. org/10.1136/bmj.j5024

Radziejewska, A., Muzsik, A., Milagro, F. I., Martínez, J. A., Chmurzynska, A. (2019). One-carbon metabolism and nonalcoholic fatty liver disease: The crosstalk between nutrients, microbiota, and genetics. Lifestyle Genom., 13(12), 53-63. http://dx.doi.org/10.1159/000504602

Ruhl, C. E., Everhart, J. E. (2005). Coffee and caffeine consumption reduce the risk of elevated serum alanine aminotransferase activity in the United States. Gastroenterology, 128(1), 24-32. http://dx.doi.org/10.1053/j. gastro.2004.09.075

Salomone, F., Volti, G. L., Vitaglione, P., Morisco, F., Fogliano, V., Zappalà, A., ..., Galvano, F. (2014). Coffee enhances the expression of chaperones and antioxidant proteins in rats with nonalcoholic fatty liver disease. Trans. Res., 163(6), 593-602. http://dx.doi. org/10.1016/j.trsl.2013.12.001

Setiawan, V. W., Wilkens, L. R., Lu, S. C., Hernandez, B. Y., Le Marchand, L., Henderson, B. E. (2015). Association of coffee intake with reduced incidence of liver cancer and death from chronic liver disease in the US multiethnic cohort. Gastroenterology, 148(1), 118-125. http:// dx.doi.org/10.1053/j.gastro.2014.10.005

Shin, J. W., Wang, J. H., Kang, J. K., Son, C. G. (2010). Experimental evidence for the protective effects of coffee against liver fibrosis in SD rats. J. Sci. Food Agric., 90(03), 450-455. http://dx.doi.org/10.1002/jsfa.3838

Shulman, G. I. (2014). Ectopic fat in insulin resistance, dyslipidemia, and cardiometabolic disease. N. Engl. J.
Med., 371(12), 1131-1141. http://dx.doi.org/10.1056/ NEJMra1011035

Stojsavljević, S., Palčić, M. G., Jukić, L. V., Duvnjak, L. S., Duvnjak, M. (2014). Adipokines and proinflammatory cytokines, the key mediators in the pathogenesis of nonalcoholic fatty liver disease. World J. Gastroent., 20(48), 18070-18091. http://dx.doi.org/10.3748/wjg.v20.i48. 18070

Townsend, S. A., Newsome, P. N. (2016). Non-alcoholic fatty liver disease in 2016. Br. Med. Bull., 119(1), 143156. http://dx.doi.org/10.1093/bmb/ldw031

Vanni, E., Bugianesi, E. (2014). Obesity and liver cancer. Clin. Liver Dis., 18(1), 191-203. http://dx.doi. org/10.1016/j.cld.2013.09.001

Vitaglione, P., Morisco, F., Mazzone, G., Amoruso, D. C., Ribecco, M. T., Romano, A., ..., D’Argenio, G. (2010). Coffee reduces liver damage in a rat model of steatohepatitis: The underlying mechanisms and the role of polyphenols and melanoidins. Hepatology, 52(5), 16521661. http://dx.doi.org/10.1002/hep.23902

WHO (2006). Acrylamide. Sixty-fourth report of the Joint FAO/WHO Expert Committee on Food Additives: Evaluation of certain food contaminants (pp. 14-25). WHO Technical Report Series - 930. Geneva: World Health Organization.

Wijarnpreecha, K., Thongprayoon, C., Ungprasert, P. (2017). Coffee consumption and risk of nonalcoholic fatty liver disease. Eur. J. Gastroenterol. Hepatol., 29(2), e8-e12. http://dx.doi.org/10.1097/MEG.0000000000000776

Word Cancer Research Fund / American Institute for Cancer Research (2018). Continuous Update Project Expert Report 2018. Diet, nutrition, psychical activity and liver cancer. Available at dietandcancerreport.org.

Yki-Järvinen, H. (2016). Diagnosis of non-alcoholic fatty liver disease (NAFLD). Diabetologia, 59(6), 1104-1111. http://dx.doi.org/10.1007/s00125-016-3944-1

Younossi, Z. M., Golabi, P., de Avila, L., Paik, J. M., Srishord, M., Fukui, N., ..., Nader, F. (2019). The global epidemiology of NAFLD and NASH in patients with type 2 diabetes: A systematic review and meta-analysis. J. Hepatol., 71(4), 793-801.

Younossi, Z. M., Koenig, A. B., Abdelatif, D., Fazel, Y., Henry, L., Wymer, M. (2016). Global epidemiology of nonalcoholic fatty liver disease. Meta-analytic assessment of prevalence, incidence, and outcomes. Hepatology, 64(1), 73-84. http://dx.doi.org/10.1002/hep.28431

Younossi, Z. M., Stepanova, M., Negro, F., Hallaji, S., Younossi, Y., Lam, B., Srishord, M. (2012). Nonalcoholic fatty liver disease in lean individuals in the United States. Medicine, 91(6), 319-327. http://dx.doi.org/10.1097/ MD.0b013e3182779d49 